\title{
Upper bounds for the Poincaré recurrence time in quantum mixed states
}

\author{
V Gimeno ${ }^{1}$ and J M Sotoca ${ }^{2}$ \\ ${ }^{1}$ Departament de Matemàtiques, Institut Universitari de Matemàtiques i Aplicacions, \\ Universitat Jaume I, Castelló, Spain \\ 2 Departament de Llenguatges i Sistemes Informàtics, Institut de Noves Tecnologies \\ de la Imatge, Universitat Jaume I, Castelló, Spain \\ E-mail: gimenov@uji.es and sotoca@uji.es
}

Received 13 July 2016, revised 27 February 2017

Accepted for publication 21 March 2017

Published 5 April 2017

\begin{abstract}
In this paper we use geometric techniques to provide upper bounds for the Poincaré recurrence time of a quantum mixed state with a discrete spectrum of energies. We obtain two types of upper bounds. One of them depends on the uncertainty in the energy or on the average of the gap of energies and extends previous results obtained for pure states. The other upper bound depends only on the number of relevant states. The first upper bound tends to zero at the classical limit, while the other bound is related with the number of relevant states and survives at the classical limit.
\end{abstract}

Keywords: Poincaré recurrence time, mixed states, fidelity

(Some figures may appear in colour only in the online journal)

\section{Introduction}

The classical Poincaré recurrence theorem states that an isolated mechanical system with a fixed finite energy and in a fixed bounded volume will return, after a sufficiently long time, close to its initial mechanical state. The Poincaré recurrence theorem follows from Liouville's theorem (see [1] for instance) due to the volume-preserving property of the Hamiltonian flux of the classical phase space. Nevertheless, the total volume of the phase space, and hence the recurrence time (length of time elapsed until the recurrence), depend on the Hamiltonian of the system.

The Poincaré recurrence theorem has counter-intuitive implications when it is considered within the context of the second law of thermodynamics. According to this law, the measure of disorder of a system will never decrease-it will either increase or stay the same. For example, considering an isolated system, if the partition separating a chamber containing a gas and a vacuum chamber is opened, after a time the gas molecules will again be collected in the first 

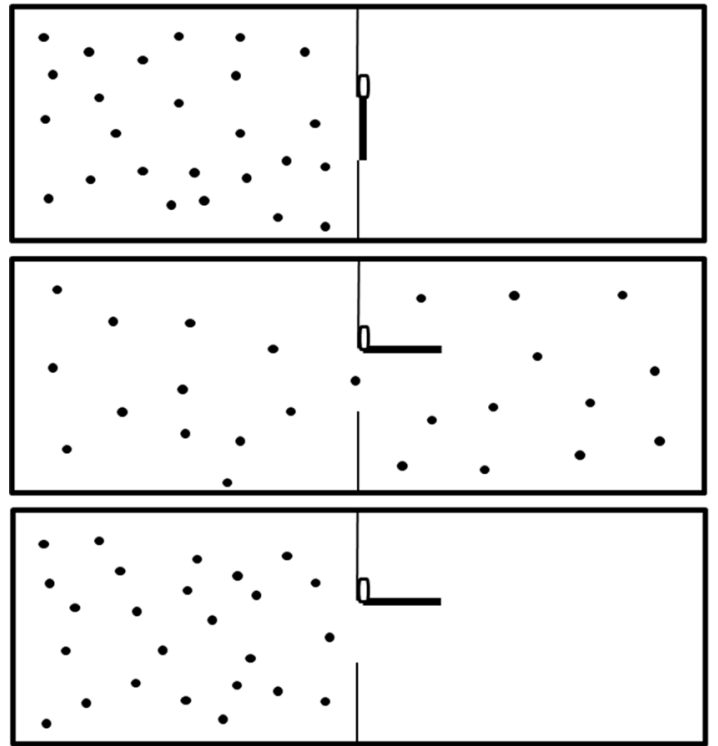

Figure 1. The 'recurrence paradox'. If we allow a gas to expand in a bounded recipient, after a sufficiently long time it will return very close to the initial position.

chamber (see figure1). This is known as the recurrence paradox and is most commonly reconciled by the claim that the amount of time that one must wait before the gas system returns to its initial state is of an order of magnitude larger than the expected life-time of the universe.

In the quantum world there is a similar equivalence to the Poincaré recurrence principle (see $[6,8,19,25,29,35])$. Thus, the quantum Poincaré recurrence appears in mixed states with time-periodic Hamiltonians for a discrete quasi-energy spectrum.

Poincaré recurrence is relevant in order to understand 'non-reversible' phenomena, such as the decoherence of a quantum system induced by the environment (see [5, 7, 27, 34, 37]), where, in order to obtain effective decoherence, a small quotient between the decoherence and the recurrence time is required. Poincare recurrence could also play an important role in the loss of information that occurs in quantum black-holes (see $[2,14,30]$ ).

Consider the evolution of an initial pure state $\left|\Psi_{0}\right\rangle \in \mathcal{H}$ of the Hilbert space $\mathcal{H}$ by the Hamiltonian operator $H$ given by the following Schrödinger equation

$$
\frac{\partial}{\partial t}|\Psi(t)\rangle=-\frac{\mathrm{i}}{\hbar} H|\Psi(t)\rangle, \quad|\Psi(0)\rangle=\left|\Psi_{0}\right\rangle .
$$

Recurrence implies in particular that for any $\epsilon>0$, there exists $t_{0}$ large enough such that

$$
\|\left|\Psi\left(t_{0}\right)\right\rangle-\left|\Psi_{0}\right\rangle \|^{2}<\epsilon
$$

Under a certain type of wave packet (see $[5,7,20,27,34,37]$ ) the recurrence time can be obtained. In [26], assuming that $\left|\Psi_{0}\right\rangle$ has a finite decomposition on the basis of eigenfunctions $\{\mid i\}\rangle$ of the Hamiltonian $H$, i.e.

$$
\left|\Psi_{0}\right\rangle=\sum_{i=1}^{n} z_{i}|i\rangle, \quad H|i\rangle=2 \pi \hbar \nu_{i}|i\rangle, \quad \|\left|\Psi_{0}\right\rangle\|=\||i\rangle \|=1,
$$


and considering that the frequencies $\left\{\nu_{i}\right\}$ are incommensurable the following estimate of the recurrence time appears

$$
t_{\mathrm{rec}} \sim \frac{1}{n^{1 / 2} \nu \sigma}
$$

where

$$
\nu=\frac{1}{n} \sum_{i=1}^{n} \nu_{i}, \quad \sigma=\frac{\pi^{\frac{n-1}{2}} R^{n-1}}{\Gamma[(n+1) / 2]}, \quad R=\frac{(n \epsilon)^{1 / 2}}{2 \pi}
$$

In [3] the authors obtain several expressions for the recurrence time, of which we highlight the following estimate

$$
t_{\mathrm{rec}} \sim \frac{1}{(n-1)^{1 / 2} \bar{\nu}_{m 1}} \Gamma\left(\frac{n}{2}\right)\left(\frac{8 \pi}{\epsilon(n-1)}\right)^{(n-2) / 2}
$$

where

$$
\bar{\nu}_{m 1}=\frac{1}{\sqrt{n-1}}\left(\sum_{m=2}^{n}\left(\nu_{m}-\nu_{1}\right)^{2}\right)^{\frac{1}{2}}
$$

We should remark here that the estimates given in equations (3) and (4) depend on the average of the energy (or the gap energy) of the quantum system.

\section{Main results}

The general case in quantum mechanics deals with mixed states. A mixed state cannot be described as a ket vector. Instead, it is described by its associated density matrix or by a density operator in a Hilbert space $\mathcal{H}$. Recall that a density matrix is a complex matrix $\rho$ that satisfies the following properties:

(i) $\rho$ is a Hermitian matrix, i.e. the matrix coincides with its conjugate transpose matrix: $\rho=\rho^{\dagger}$.

(ii) $\rho$ is positive $\rho \geqslant 0$. This means that any eigenvalue of $A$ is non-negative.

(iii) $\rho$ is normalized by the $\operatorname{trace} \operatorname{tr}(\rho)=1$.

The temporal evolution of a mixed state $\rho_{0}$ is given by von-Neumann's law

$$
\dot{\rho}(t)=-\frac{\mathrm{i}}{\hbar}[H, \rho(t)], \quad \rho(0)=\rho_{0} .
$$

The probability of a transition from the mixed state $\rho$ to the mixed state $\sigma$ is given now by the fidelity $F(\rho, \sigma)$

$$
F(\rho, \sigma):=\operatorname{tr}[\sqrt{\sqrt{\rho} \sigma \sqrt{\rho}}] .
$$

We define the recurrence time as the first time $\rho(t)$ returns close to $\rho_{0}$ after passing the first exit time. And we define the first exit time as the time required for $\rho(t)$ to escape from $\rho_{0}$. More precisely, at $t=0, \rho(0)=\rho_{0}$ and obviously $F\left(\rho_{0}, \rho(0)\right)=1$. Now, set $0<\epsilon<1$, therefore by continuity of $F$, for any $t>0$ that is small enough, $F\left(\rho_{0}, \rho(t)\right)>\epsilon$ (the mixed state $\rho(t)$ is close to $\rho_{0}$, in the sense that the fidelity between $\rho(t)$ and $\rho_{0}$ is greater than $\epsilon$ ). Without loss of 
generality we can assume ${ }^{3}$ that the fidelity between $\rho(t)$ and $\rho_{0}$ remains greater than $\epsilon$ up to the first exit time $t_{\text {exit }}(\epsilon)$, where

$F\left(\rho_{0}, \rho\left(t_{\text {exit }}(\epsilon)\right)\right)=\epsilon, \quad F\left(\rho_{0}, \rho\left(t_{\text {exit }}(\epsilon)-\delta\right)\right)>\epsilon, \quad F\left(\rho_{0}, \rho\left(t_{\text {exit }}(\epsilon)+\delta\right)\right)<\epsilon$,

for any $0<\delta \ll 1$. Hence, immediately after the first exit time, the fidelity between $\rho(t)$ and $\rho_{0}$ is less than $\epsilon$. We define the recurrence time $t_{\mathrm{rec}}(\epsilon)$ as the first time after $t_{\text {exit }}(\epsilon)$ such that

$$
F\left(\rho_{0}, \rho\left(t_{\mathrm{rec}}\right)\right)=\epsilon .
$$

Stated otherwise, the recurrence time $t_{\mathrm{rec}}(\epsilon)$ is the first time when the mixed state returns close to $\rho_{0}$, in the sense that the fidelity between $\rho\left(t_{\text {rec }}(\epsilon)\right)$ and $\rho_{0}$ takes the value $\epsilon$ again. Here we must stress the importance of defining the recurrence time as greater than the first exit time to take into account the recurrent feature of the movement. Otherwise, condition (5) is not related with any recurrent phenomenon but with an escape ratio.

In this paper we shall assume that the spectrum of the Hamiltonian is discrete, with orthonormal eigenstates $\{\mid k\}\rangle_{k=1}^{\infty},\left(H|k\rangle=E_{k}|k\rangle,\langle k \mid j\rangle=\delta_{k, j}\right)$. The relevant Hilbert space to describe the evolution of $\rho_{0}$ is the span of the energy eigenstates on which $\rho$ has support. The dimension of that Hilbert space is the the number of energy eigenvalues $E_{k}$ with $p_{k}:=\left\langle k\left|\rho_{0}\right| k\right\rangle$ non-zero.

Our results deal with mixed states with a discrete and finite spectrum (section 2.1) and with mixed states with a discrete and non-finite spectrum (section 2.2). The proofs of the theorems in sections 2.1 and 2.2 will be performed in section 3 .

\subsection{Mixed states with discrete and finite spectrum}

For a mixed state with a discrete and finite spectrum we can provide the following theorem with estimates of the recurrence time, similar to that shown in expressions (3) and (4).

Theorem 1. Let $\rho_{0}$ be a mixed state of the Hilbert space $\mathcal{H}$ of finite dimension $n=\operatorname{dim}(\mathcal{H})$. Let $\rho(t)$ denote the unitary evolution given by the Hamiltonian $H$, i.e.

$$
\dot{\rho}(t)=-\frac{\mathrm{i}}{\hbar}[H, \rho(t)], \quad \rho(0)=\rho_{0} .
$$

Let $\{|k\rangle\}$ be a basis of orthonormal eigenstates of the Hamiltonian (i.e. $H|k\rangle=E_{k}|k\rangle$ and $\left.\langle j \mid k\rangle=\delta_{j, k}\right)$. Then

(i) If the initial mixed state $\rho_{0}$ has non-zero uncertainty in the energy $\triangle E_{\rho_{0}} \neq 0$ (with $\triangle E_{\rho_{0}}=\sqrt{\left.\operatorname{tr}\left(H^{2} \rho_{0}\right)-\operatorname{tr}\left(H \rho_{0}\right)^{2}\right)}$ and for some $\epsilon \geqslant 1-\frac{\pi^{2} \min _{k}\left\{\operatorname{tr}\left(\rho_{0}|k\rangle\langle k|\right)\right\}}{2}$

$$
t_{\text {exit }}(\epsilon) \leqslant \hbar \frac{\pi \cdot \min _{k}\left\{\sqrt{\operatorname{tr}\left(\rho_{0}|k\rangle\langle k|\right)}\right\}}{\triangle E_{\rho_{0}}}
$$

Then,

$$
t_{\mathrm{rec}}(\epsilon) \leqslant \hbar \frac{c_{n}}{(1-\epsilon)^{\frac{n-1}{2}}} \cdot \frac{\prod_{k} \sqrt{\operatorname{tr}\left(\rho_{0}|k\rangle\langle k|\right)}}{\triangle E_{\rho_{0}}}
$$

(ii) If $\operatorname{tr}\left(\rho_{0}\left(H-E_{1} I\right)^{2}\right) \neq 0$, and for some $\epsilon \geqslant 1-\frac{\pi^{2} \min _{k}\left\{\operatorname{tr}\left(\rho_{0}|k\rangle\langle k|\right)\right\}}{2}$

\footnotetext{
${ }^{3}$ if there is no such first exit time, to obtain upper bounds for the recurrence time is trivial.
} 


$$
t_{\text {exit }}(\epsilon) \leqslant \hbar \frac{\pi \cdot \min _{k}\left\{\sqrt{\operatorname{tr}\left(\rho_{0}|k\rangle\langle k|\right)}\right\}}{\sqrt{\operatorname{tr}\left(\rho_{0}\left(H-E_{1} I\right)^{2}\right)}}
$$

Then,

$$
t_{\mathrm{rec}}(\epsilon) \leqslant \hbar \frac{c_{n}}{(1-\epsilon)^{\frac{n-1}{2}}} \cdot \frac{\prod_{k} \sqrt{\operatorname{tr}\left(\rho_{0}|k\rangle\langle k|\right)}}{\sqrt{\operatorname{tr}\left(\rho_{0}\left(H-E_{1} I\right)^{2}\right)}}
$$

with $c_{n}=(n-1) \Gamma\left(\frac{n-1}{2}\right) 2^{\frac{3 n-3}{2}} \pi^{\frac{n+1}{2}}$

Note that since $t_{\text {exit }}(\epsilon) \rightarrow 0$ when $\epsilon \rightarrow 1$, then it is always possible to choose $\epsilon$ close enough to 1 such that inequalities (6) or (8) are satisfied.

Estimates (7) and (9) can be simplified by using the arithmetic-mean geometric-mean inequality

$$
\begin{aligned}
\prod_{k=1}^{n} \sqrt{\operatorname{tr}\left(\rho_{0}|k\rangle\langle k|\right)}= & {\left[\left(\prod_{k=1}^{n} \operatorname{tr}\left(\rho_{0}|k\rangle\langle k|\right)\right)^{\frac{1}{n}}\right]^{\frac{n}{2}} } \\
& \leqslant\left[\frac{\sum_{k=1}^{n} \operatorname{tr}\left(\rho_{0}|k\rangle\langle k|\right)}{n}\right]^{\frac{n}{2}}=\frac{1}{n^{\frac{n}{2}}}
\end{aligned}
$$

Therefore under the same hypothesis as the above theorem we can state

$$
t_{\mathrm{rec}}(\epsilon) \leqslant\left(\frac{c_{n}}{(1-\epsilon)^{\frac{n-1}{2}} n^{\frac{n}{2}}}\right) \frac{\hbar}{\triangle E_{\rho_{0}}}
$$

or

$$
t_{\text {rec }}(\epsilon) \leqslant\left(\frac{c_{n}}{(1-\epsilon)^{\frac{n-1}{2}} n^{\frac{n}{2}}}\right) \frac{\hbar}{\sqrt{\operatorname{tr}\left(\rho_{0}\left(H-E_{1} I\right)^{2}\right)}}
$$

In the next theorem we can establish a relation between $t_{\text {exit }}(\epsilon)$ and $t_{\text {rec }}(\epsilon)$

Theorem 2. Let $\rho_{0}$ be a mixed state of the Hilbert space $\mathcal{H}$ of finite dimension $n=\operatorname{dim}(\mathcal{H})$. Let $\rho(t)$ denote the unitary evolution given by the Hamiltonian $H$, i.e.

$$
\dot{\rho}(t)=-\frac{\mathrm{i}}{\hbar}[H, \rho(t)], \quad \rho(0)=\rho_{0} .
$$

Let $\{|k\rangle\}$ be a basis of orthonormal eigenstates of the Hamiltonian (i.e. $H|k\rangle=E_{k}|k\rangle$ and $\left.\langle j \mid k\rangle=\delta_{j, k}\right)$. Then if the initial mixed state $\rho_{0}$ has non-zero uncertainty in the energy $\triangle E_{\rho_{0}} \neq 0$ (with $\triangle E_{\rho_{0}}=\sqrt{\operatorname{tr}\left(H^{2} \rho_{0}\right)-\operatorname{tr}\left(H \rho_{0}\right)^{2}}$ ) and for some $\epsilon \geqslant 1-\frac{\pi^{2} \min _{k}\left\{\operatorname{tr}\left(\rho_{0}|k\rangle\langle k|\right)\right\}}{2}$

$$
t_{\text {exit }}(\epsilon) \leqslant \hbar \frac{\pi \cdot \min _{k}\left\{\sqrt{\operatorname{tr}\left(\rho_{0}|k\rangle\langle k|\right)}\right\}}{\triangle E_{\rho_{0}}}
$$


Then,

$$
t_{\text {rec }}(\epsilon) \leqslant \frac{c_{n}}{(1-\epsilon)^{\frac{n-1}{2}}} \frac{\prod_{k} \sqrt{\operatorname{tr}\left(\rho_{0}|k\rangle\langle k|\right)}}{\pi \cdot \min _{k}\left\{\sqrt{\operatorname{tr}\left(\rho_{0}|k\rangle\langle k|\right)}\right\}} \cdot t_{\text {exit }}(\epsilon)
$$

In the case of a finite number of states there is an upper bound for the recurrence time that does not depend on the specific Hamiltonian. In fact we can state

Theorem 3. Let $\rho_{0}$ be a mixed state of the Hilbert space $\mathcal{H}$ of finite dimension $n=\operatorname{dim}(\mathcal{H})$. Let $\rho(t)$ denote the unitary evolution given by the Hamiltonian $H$, i.e.

$$
\dot{\rho}(t)=-\frac{\mathrm{i}}{\hbar}[H, \rho(t)], \quad \rho(0)=\rho_{0} .
$$

Then,

$$
t_{\mathrm{rec}}(\epsilon) \leqslant C(n, \epsilon) t_{\mathrm{exit}}(\epsilon)
$$

where the constant $C(n, \epsilon):=\sqrt{\pi} \frac{\Gamma\left(n^{2}\right)}{\Gamma\left(n^{2}+\frac{1}{2}\right)} \frac{1}{\int_{0}^{\frac{\sqrt{2-2 \epsilon}}{2}} \sin ^{2 n^{2}-2}(s) \mathrm{d} s}$ depends only on $\epsilon$ and on the dimension of the Hilbert space.

Then, the smaller the first exit time is, the smaller the recurrence time will be. In order to understand this behavior let us point out the following example in the case of $n=2$, where the motion is always strictly periodic. Let $\{|j\rangle\}_{j=1}^{\infty}$ be an orthonormal basis for the Hamiltonian $\left(H|j\rangle=E_{j}|j\rangle\right.$, and $\langle j \mid k\rangle=\delta_{j, k}$, let $|\Psi\rangle=c_{1}|1\rangle+c_{2}|2\rangle$ be a normalized pure state $\left(\left|c_{1}\right|^{2}+\left|c_{2}\right|^{2}=1\right)$. Thus, the associated density matrix is given by

$$
\rho_{0}=|\Psi\rangle\langle\Psi|=\left(\begin{array}{ll}
\left|c_{1}\right|^{2} & c_{1} c_{2}^{*} \\
c_{1}^{*} c_{2} & \left|c_{2}\right|^{2}
\end{array}\right)
$$

and the solution to $\dot{\rho}(t)=-\frac{\mathrm{i}}{\hbar}[H, \rho(t)]$ with $\rho(0)=\rho_{0}$ is given by

$$
\rho(t)=|\Psi(t)\rangle\langle\Psi(t)|=\left(\begin{array}{cc}
\left|c_{1}\right|^{2} & c_{1} c_{2}^{*} \mathrm{e}^{-\mathrm{i} w t} \\
c_{1}^{*} c_{2} \mathrm{e}^{-\mathrm{i} w t} & \left|c_{2}\right|^{2}
\end{array}\right), \quad w:=\frac{E_{2}-E_{1}}{\hbar} .
$$

The fidelity between $\rho(t)$ and $\rho_{0}$ is therefore

$$
F\left(\rho_{0}, \rho(t)\right)=|\langle\Psi(t) \mid \Psi\rangle|=1-2\left|c_{1}\right|^{2}\left|c_{2}\right|^{2}+2\left|c_{1}\right|^{2}\left|c_{2}\right|^{2} \cos (w t)
$$

where the fidelity between $\rho_{0}$ and $\rho(t)$ starts with the value of 1 for $t=0$ and decreases to its minimum of $1-4\left|c_{1}\right|^{2}\left|c_{2}\right|^{2}$ at $t=\pi / w$. Hence, for any $\epsilon>1-4\left|c_{1}\right|^{2}\left|c_{2}\right|^{2}$ there exists a time $t_{\text {exit }}(\epsilon)$ such that $F\left(\rho_{0}, \rho\left(t_{\text {exit }}(\epsilon)\right)\right)=\epsilon$. In fact,

$$
t_{\text {exit }}(\epsilon)=\frac{1}{w} \arccos \left(1-\frac{1-\epsilon}{2\left|c_{1}\right|^{2}\left|c_{2}\right|^{2}}\right)
$$

Immediately after $t_{\text {exit }}(\epsilon)$ the fidelity between $\rho_{0}$ and $\rho(t)$ is less than $\epsilon$. But at time $t_{\text {rec }}(\epsilon)=\frac{2 \pi}{w}-t_{\text {exit }}(\epsilon)$, the fidelity between $\rho_{0}$ and $\rho(t)$ is $\epsilon$ again. Observe that

$t_{\mathrm{rec}}(\epsilon)=\left(\frac{2 \pi}{\arccos \left(1-\frac{1-\epsilon}{2\left|c_{1}\right|^{2}\left|c_{2}\right|^{2}}\right)}-1\right) t_{\mathrm{exit}}(\epsilon) \leqslant\left(\frac{2 \pi}{\arccos (2 \epsilon-1)}-1\right) t_{\text {exit }}(\epsilon)$ 
By theorem 3 and inequality (14) we know that this kind of behavior is not only true for $n=2$ and pure states but also for any mixed state of a finite spectrum. We must notice here that $\frac{2 \pi}{\arccos (2 \epsilon-1)}-1 \leqslant C(2, \epsilon)$ because all the hypotheses of theorem 3 are fulfilled. Although the constant $C(n, \epsilon)$ and inequality (14) are not sharp for the case of $n=2$ and pure states, inequality (14) remains true in the general case of mixed states and $n \in \mathbb{N}$.

Remark 1. After the recurrence time, $\rho(t)$ returns infinitely many times close to $\rho_{0}$. Namely, for an arbitrary $\epsilon \in[0,1)$ the inequality

$$
F\left(\rho(T), \rho_{0}\right)>\epsilon
$$

is satisfied by infinitely many values of $T$, these values being spread over the whole range from 0 to $\infty$. Indeed, in proposition 4 we prove that for any $s>0$ there exist $T$ with

$$
s \leqslant T \leqslant C(n, \epsilon) s
$$

such that (15) holds.

\subsection{Mixed states with discrete but non-finite spectrum}

In a similar way to how the problem is set out in [25], we consider $\rho(t)$ the density matrix of a system for a set of discrete stationary states, with energy levels $E_{k}, k=0,1,2, \cdots$, where some of them may have the same value if they are degenerate. In energy representation, the matrix elements are defined as

$$
\rho_{k k^{\prime}}(t)=\left\langle k|\rho(t)| k^{\prime}\right\rangle .
$$

Let $T_{k}=|k\rangle\langle k|$ be the projection operator onto the $k$ th stationary state, then

$$
\rho^{k k^{\prime}}(t)=T_{k} \rho(t) T_{k^{\prime}},
$$

is the matrix whose energy representation has only one non-zero element, equal to $\rho_{k k^{\prime}}(t)$ and located at $\left(k, k^{\prime}\right)$. These matrices are orthogonal in density space

$$
\operatorname{tr}\left(\rho^{k k^{\prime}}(t)^{\dagger} \rho^{k^{\prime \prime} k^{\prime \prime \prime}}(t)\right)=\delta_{k k^{\prime \prime}} \delta_{k^{\prime} k^{\prime \prime \prime}}\left|\rho_{k k^{\prime}}(t)\right|^{2},
$$

and

$$
\begin{aligned}
\rho(t) & =\sum_{k=0}^{\infty} \sum_{k^{\prime}=0}^{\infty} \rho^{k k^{\prime}}(t) \\
& =\sum_{k=0}^{\infty} \sum_{k^{\prime}=0}^{\infty} \rho^{k k^{\prime}}(0) \mathrm{e}^{\mathrm{i} \omega_{k k^{\prime}} t},
\end{aligned}
$$

where $\omega_{k k^{\prime}}=\frac{E_{k^{\prime}}-E_{k}}{\hbar}$. Now, regard the finite sum

$$
\sigma^{N}(t)=\sum_{k=0}^{N} \sum_{k^{\prime}=0}^{N} \rho^{k k^{\prime}}(t),
$$

as an approximation to $\rho(t)$. Then, the squared error is 


$$
\begin{aligned}
\left\|\rho(t)-\sigma^{N}(t)\right\|_{2}^{2} & =\left\|\sum_{k=N+1}^{\infty} \sum_{k^{\prime}=N+1}^{\infty} \rho^{k k^{\prime}}(t)\right\|_{2}^{2} \\
& =\sum_{k=N+1}^{\infty} \sum_{k^{\prime}=N+1}^{\infty}\left\|\rho^{k k^{\prime}}(t)\right\|_{2}^{2} \\
& =\sum_{k=N+1}^{\infty} \sum_{k^{\prime}=N+1}^{\infty}\left\|\rho^{k k^{\prime}}(0)\right\|_{2}^{2} .
\end{aligned}
$$

Here \|\|$_{2}$ indicates the Frobenius norm, i.e. $\|A\|_{2}=\sqrt{\operatorname{tr}\left(A^{\dagger} A\right)}$. The second equality is achieved from the orthogonality of $\rho^{k k^{\prime}}$. Because the error is not time-dependent, $\sigma^{N}(t)$ converges uniformly to $\rho(t)$ (in the \|\|$_{2}$-norm sense). Let us denote by $\delta_{N}$ the time-independent quantity

$$
\delta_{N}:=\left\|\rho(t)-\sigma^{N}(t)\right\|_{2}^{2}=\left\|\rho(0)-\sigma^{N}(0)\right\|_{2}^{2}
$$

So, $\rho(t)$ can be approximated by $\sigma^{N}(t)$ in the sense that $\delta_{N} \rightarrow 0$ when $N \rightarrow \infty$. Thereupon, we shall say that the mixed state $\rho$ has $N$ relevant states with error term of $\delta_{N}$.

Observe that the trace of $\sigma^{N}(t)$ is time-independent because

$$
\operatorname{tr}\left(\sigma^{N}(t)\right)=\operatorname{tr}\left(\mathrm{e}^{\frac{-i H}{\hbar} t} \sigma^{N}(0) \mathrm{e}^{\frac{i H}{\hbar} t}\right)=\operatorname{tr}\left(\sigma^{N}(0)\right)
$$

We can define

$$
\widetilde{\sigma}^{N}(t):=\frac{1}{\operatorname{tr}\left(\sigma^{N}(0)\right)^{\frac{1}{N}}} \sigma^{N}(t)
$$

then

$$
\widetilde{\sigma}^{N}(t)=\mathrm{e}^{\frac{-\mathrm{i} H}{\hbar} t} \widetilde{\sigma}^{N}(0) \mathrm{e}^{\frac{\mathrm{i} H}{\hbar} t}, \quad \operatorname{tr}\left(\widetilde{\sigma}^{N}(t)\right)=1 .
$$

Hence, $\widetilde{\sigma}^{N}$ fulfills the hypotheses of theorems $1-3$. But by using the triangular inequality

$$
\begin{aligned}
\|\rho(t)-\rho(0)\|_{2} & \leqslant\left\|\rho(t)-\sigma^{N}(t)\right\|_{2} \\
& +\left\|\sigma^{N}(t)-\sigma^{N}(0)\right\|_{2}+\left\|\sigma^{N}(0)-\rho(0)\right\|_{2} \\
& \leqslant 2 \sqrt{\delta_{N}}+\left\|\sigma^{N}(t)-\sigma^{N}(0)\right\|_{2}
\end{aligned}
$$

Then,

$$
\|\rho(t)-\rho(0)\|_{2} \leqslant 2 \sqrt{\delta_{N}}+\left\|\widetilde{\sigma}^{N}(t)-\widetilde{\sigma}^{N}(0)\right\|_{2} \operatorname{tr}\left(\sigma^{N}(0)\right)^{\frac{1}{N}}
$$

Observe that

$$
P_{N}:=\operatorname{tr}\left(\sigma^{N}(0)\right)=\sum_{k=1}^{N} \rho_{k, k}(0)=\sum_{k=1}^{N}\langle k|\rho(0)| k\rangle=\sum_{k=1}^{N} \operatorname{tr}(\rho(0)|k\rangle\langle k|)
$$

is the total probability of $\rho$ being in one of the relevant $N$ states. By the Fuchs-van de Graaf inequalities (see [36] for instance)

$$
F\left(\widetilde{\sigma}^{N}(0), \widetilde{\sigma}^{N}(t)\right) \leqslant \sqrt{1-\frac{1}{4}\left\|\widetilde{\sigma}^{N}(t)-\widetilde{\sigma}^{N}(0)\right\|_{1}^{2}}
$$

where here \|\|$_{1}$ denotes the trace norm (i.e. $\|A\|_{1}=\operatorname{tr}\left(\sqrt{A^{\dagger} A}\right)$ ). But since \|\|$_{1} \geqslant\|\|_{2}$, 


$$
\left\|\widetilde{\sigma}^{N}(t)-\widetilde{\sigma}^{N}(0)\right\|_{2} \leqslant 2 \sqrt{1-F^{2}\left(\widetilde{\sigma}^{N}(0), \tilde{\sigma}^{N}(t)\right)}
$$

Therefore

$$
\|\rho(t)-\rho(0)\|_{2} \leqslant 2 \sqrt{\delta_{N}}+2 \operatorname{tr}\left(\sigma^{N}(0)\right)^{\frac{1}{N}} \sqrt{1-F^{2}\left(\widetilde{\sigma}^{N}(0), \tilde{\sigma}^{N}(t)\right)}
$$

The evolution of $\rho(t)$ is governed by the evolution of $\widetilde{\sigma}^{N}(t)$ in the following sense, when $\widetilde{\sigma}^{N}(t)$ attains the recurrence time $t_{\text {rec }}^{\widetilde{\sigma}^{N}}(\epsilon), \rho$ is close to $\rho_{0}$ in the sense that

$$
\left\|\rho\left(t_{\text {rec }}^{\widetilde{\sigma}^{N}}(\epsilon)\right)-\rho(0)\right\|_{2} \leqslant 2 \sqrt{\delta_{N}}+2 \operatorname{tr}\left(\sigma^{N}(0)\right)^{\frac{1}{N}} \sqrt{1-\epsilon^{2}}
$$

where $\delta_{N} \rightarrow 0$ and $\operatorname{tr}\left(\sigma^{N}(0)\right)^{\frac{1}{N}} \rightarrow 1$ when $N \rightarrow \infty$. In consequence, we can use the upper bounds for the recurrence time $\widetilde{\sigma}^{N}$ of the previous section to obtain the upper bounds for the recurrence time of $\rho$.

\section{Proof of theorems}

To prove the upper bounds for the recurrence time we will make use of geometric techniques. The key idea is to confine the movement in a space of finite total volume and to show that certain geometric domains with known volume are not intersecting up to the recurrence time. We therefore obtain the upper bound for the recurrence time as the quotient of the total volume and the volume of the domain.

The specific domain used in the proof of theorems 1 and 2 is a geodesic ball and the total space is a $2 n^{2}-1$-sphere, while the specific domain used to prove theorem 3 is a tube around a geodesic curve and the total space is a $n$-torus. Because the $n$-torus is a submanifold of the $2 n^{2}-1$-sphere, the first part of this (section 3.1) deals with the description of the geometry of such a sphere and the proof of theorem 3. In the last part of this (appendix A.2) we will prove proposition 6, which implies theorems 1 and 2.

\subsection{Geometry of the mixed states space. Proof of theorem 3}

In this part of the paper we always assume that $\operatorname{dim}(\mathcal{H})=n<\infty, \mathcal{H}$ being a vector space in the complex field $\left(\mathcal{H}=\mathbb{C}^{n}\right)$. Let us denote by $\mathcal{D}$ the space of density matrices and let us denote by

$$
\mathcal{P}^{+}:=\{\rho \in \mathcal{D}: \rho>0\}
$$

Let us consider the following sphere

$$
\widetilde{S}:=\left\{W \in M_{n}(\mathbb{C}): \operatorname{tr}\left(W W^{\dagger}\right)=1\right\}
$$

where $M_{n}(\mathbb{C})$ is the vector space of complex matrices with $n$ rows and $n$ columns. And let us define the open dense set of $\widetilde{S}$,

$$
S:=\widetilde{S} \cap \operatorname{GL}(n, \mathbb{C})
$$

where $\mathrm{GL}(n, \mathbb{C})$ is the general linear group over the field of complex numbers. Since $M_{n}(\mathbb{C})$ is a vector space, the tangent space $T_{p} M_{n}(\mathbb{C})$ at $p \in M_{n}(\mathbb{C})$ can be identified with $M_{n}(\mathbb{C})$ itself. Moreover, we will denote by $g$ the Euclidean metric in $M_{n}(\mathbb{C})$, namely,

$$
g(X, Y)=\frac{1}{2} \operatorname{tr}\left(X^{\dagger} Y+X Y^{\dagger}\right)
$$


and we will also denote by $g$ the restriction of the above metric tensor to $\widetilde{S}$. To prove theorem 3 we are going to prove that there is an isometry of $\widetilde{S}$ related with the Hamiltonian and by volume conservation we shall estimate the upper bounds for the recurrence time. Indeed we can obtain the following proposition

Proposition 4. Let $\rho_{0}$ be a mixed state of the Hilbert space $\mathcal{H}$ of finite dimension $n=\operatorname{dim}(\mathcal{H})$. Let $\rho(t)$ denote the unitary evolution given by the Hamiltonian H, i.e.

$$
\dot{\rho}(t)=-\frac{\mathrm{i}}{\hbar}[H, \rho(t)], \quad \rho(0)=\rho_{0} .
$$

Then, for any $s>0$ and any $\epsilon \in[0,1)$ there exists a time $t_{s}(\epsilon)$ such that

$$
F\left(\rho_{0}, \rho\left(t_{s}(\epsilon)\right)\right) \geqslant \epsilon,
$$

with $t_{s}(\epsilon)=j \cdot s, j \in \mathbb{N}$ and such that

$$
1 \leqslant j \leqslant \sqrt{\pi} \frac{\Gamma\left(n^{2}\right)}{\Gamma\left(n^{2}+\frac{1}{2}\right)} \frac{1}{\int_{0}^{\frac{\sqrt{2-2 \epsilon}}{2}} \sin ^{2 n^{2}-2}(s) \mathrm{d} s} .
$$

Theorem 3 follows immediately by setting $s=t_{\text {exit }}(\epsilon)$ in the above proposition.

Proof of proposition 4. We are going to prove that the map $T_{t}: \widetilde{S} \rightarrow \widetilde{S}$ given by

$$
T_{t}(x)=\mathrm{e}^{-\frac{\mathrm{i} H t}{\hbar}} x
$$

is an isometry of $\widetilde{S}$. Suppose that we have two vectors $X, Y \in T_{x} \widetilde{S}$ then we need to check whether

$$
g(X, Y)=g\left(\mathrm{~d} T_{t}(X), \mathrm{d} T_{t}(Y)\right)
$$

In order to do so, consider the following two curves $\gamma_{X}: \mathbb{R} \rightarrow \widetilde{S}$ and $\gamma_{Y}: \mathbb{R} \rightarrow \widetilde{S}$, such that

$$
\gamma_{X}(0)=\gamma_{Y}(0)=x, \quad \dot{\gamma}_{X}(0)=X, \quad \dot{\gamma}_{Y}(0)=Y .
$$

Then,

$$
\mathrm{d} T_{t}(X)=\left.\frac{\mathrm{d}}{\mathrm{d} s} T_{t}\left(\gamma_{X}(s)\right)\right|_{s=0}=\left.\frac{d}{\mathrm{~d} s}\left(\mathrm{e}^{-\frac{\mathrm{i} H t}{\hbar}} \gamma_{X}(s)\right)\right|_{s=0}=\mathrm{e}^{-\frac{\mathrm{i} H t}{\hbar}} X
$$

For $\mathrm{d} T_{t}(Y)$ we can obtain in an analogous way that $\mathrm{d} T_{t}(Y)=\mathrm{e}^{-\frac{\mathrm{i} H t}{\hbar}} Y$. Hence,

$$
\begin{aligned}
g\left(\mathrm{~d} T_{t}(X), \mathrm{d} T_{t}(Y)\right) & =g\left(\mathrm{e}^{-\frac{\mathrm{i} H t}{\hbar}} X, \mathrm{e}^{-\frac{\mathrm{i} H t}{\hbar}} Y\right) \\
& =\frac{1}{2} \operatorname{tr}\left(\left(\mathrm{e}^{-\frac{\mathrm{i} H t}{\hbar}} X\right)^{\dagger} \mathrm{e}^{-\frac{\mathrm{i} H t}{\hbar}} Y+\left(\mathrm{e}^{-\frac{\mathrm{i} H t}{\hbar}} Y\right)^{\dagger} \mathrm{e}^{-\frac{\mathrm{i} H t}{\hbar}} X\right) \\
& =\frac{1}{2} \operatorname{tr}\left(X^{\dagger} Y+Y^{\dagger} X\right)=g(X, Y)
\end{aligned}
$$

This is what had to be proved. Because $T_{t}$ is an isometry in a metric space of finite measure, and applying theorem 7 to $\widetilde{S}$, and taking into account the volume of a geodesic ball in $\mathbb{S}^{2 n^{2}-1}$ (see equation (A.1)), we conclude that

Proposition 5. For any $A \in \widetilde{S}$ and any $t \geqslant 0$, and any $r>0$ there exists $N_{r} \in \mathbb{N}$ such that

$$
\operatorname{dist}_{\widetilde{S}}\left(A, T_{N_{r} t}(A)\right) \leqslant r
$$


where $\operatorname{dist}_{\widetilde{S}}(\cdot, \cdot)$ is the distance function on $\widetilde{S}$. Furthermore, $N_{r}$ satisfies

$$
1 \leqslant N_{r} \leqslant \frac{\mu(\widetilde{S})}{\mu\left(B_{r / 2}\right)}
$$

with

$$
\frac{\mu(\widetilde{S})}{\mu\left(B_{r / 2}\right)}=\frac{\int_{0}^{\pi} \sin ^{2 n^{2}-2}(s) \mathrm{d} s}{\int_{0}^{r / 2} \sin ^{2 n^{2}-2}(s) \mathrm{d} s}
$$

Following the results obtained by Uhlmann [31-33], Bengtsson [4], Chruściński [10], and Dąbrowski [11-13], we can consider the following principal fiber bundle

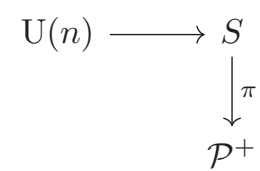

where the projection $\pi: S \rightarrow \mathcal{P}^{+}$is given by $\pi(A)=A A^{\dagger}$ and $\mathrm{U}(n)$ is the unitary group. The group $\mathrm{U}(n)$ acts on $S$ by right multiplication, i.e. $(u, A) \rightarrow A u$ for $A \in S$ and $u \in \mathrm{U}(n)$. Bearing in mind that since $S$ is an open and dense subset of $\widetilde{S}$, we can endow $S$ with the restriction of the metric $g$ of $\widetilde{S}$. Hereafter, by using this metric structure the following fiber bundle

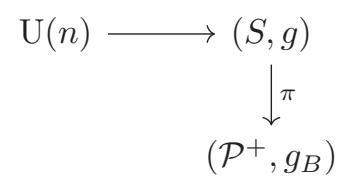

becomes a Riemannian submersion, where $g_{B}$ is the Bures metric in $\mathcal{P}^{+}$and $\mathrm{U}(n)$ acts by isometries on $S$. Notice, moreover, that

$$
\frac{\partial}{\partial t}\left(\pi \circ T_{t}(A)\right)=-\frac{\mathrm{i}}{\hbar}\left[H, \pi \circ T_{t}(A)\right]
$$

Also by using the globally defined section $s: \mathcal{P}^{+} \rightarrow S$ given by $s(\rho)=\sqrt{\rho}$ (with a particular choice of the square root branch), the general solution of the von Neumann equation

$$
\frac{\partial}{\partial t}(\rho(t))=-\frac{\mathrm{i}}{\hbar}[H, \rho(t)], \quad \rho(0)=\rho_{0}
$$

can be obtained as

$$
\rho(t)=\pi\left(T_{t}\left(s\left(\rho_{0}\right)\right)\right)
$$

Whereas $\operatorname{dist}_{S}=\operatorname{dist}_{\widetilde{S}}$ and taking into account that $\pi$ is a Riemannian submersion, then

$$
\operatorname{dist}_{\text {Bures }}\left(\pi(A), \pi\left(T_{N_{r} t}(A)\right)\right) \leqslant \operatorname{dist}_{S}\left(A, T_{N_{r} t}(A)\right) \leqslant r
$$

Hence, the theorem follows by using the above inequality for the particular case (see equation (19)) of $A=s\left(\rho_{0}\right)$, because

$$
\sqrt{2-2 F\left(\rho\left(N_{r} t\right), \rho_{0}\right)}=\operatorname{dist}_{\text {Bures }}\left(\rho(t), \rho_{0}\right) \leqslant r
$$


and we can set

$$
\epsilon=1-\frac{r^{2}}{2}, \quad j=N_{r},
$$

then

$$
F\left(\rho(j \cdot t), \rho_{0}\right) \geqslant \epsilon
$$

\subsection{Proof of theorems 1 and 2}

The proofs of theorems 1 and 2 are a consequence of the following proposition

Proposition 6. Let $\rho_{0}$ be a mixed state of the Hilbert space $\mathcal{H}$ of finite dimension $n=\operatorname{dim}(\mathcal{H})$. Let $\rho(t)$ denote the unitary evolution given by the Hamiltonian H, i.e.

$$
\dot{\rho}(t)=-\frac{\mathrm{i}}{\hbar}[H, \rho(t)], \quad \rho(0)=\rho_{0} .
$$

Suppose that for some $\lambda \in \mathbb{R}$, and for some $\epsilon \geqslant 1-\frac{\pi^{2} \min _{k}\left\{\operatorname{tr}\left(\rho_{0}|k\rangle\langle k|\right)\right\}}{2}$

$$
t_{\text {exit }}(\epsilon) \leqslant \hbar \frac{\pi \cdot \min _{k}\left\{\sqrt{\operatorname{tr}\left(\rho_{0}|k\rangle\langle k|\right)}\right\}}{\sqrt{\left(E_{1}-\lambda\right)^{2} \operatorname{tr}\left(\rho_{0}|1\rangle\langle 1|\right)+\cdots+\left(E_{n}-\lambda\right)^{2} \operatorname{tr}\left(\rho_{0}|n\rangle\langle n|\right)}}
$$

Then,

$$
t_{\text {rec }}(\epsilon) \leqslant \hbar \frac{c_{n}}{(1-\epsilon)^{\frac{n-1}{2}}} \cdot \frac{\prod_{k} \sqrt{\operatorname{tr}\left(\rho_{0}|k\rangle\langle k|\right)}}{\sqrt{\left(E_{1}-\lambda\right)^{2} \operatorname{tr}\left(\rho_{0}|1\rangle\langle 1|\right)+\cdots+\left(E_{n}-\lambda\right)^{2} \operatorname{tr}\left(\rho_{0}|n\rangle\langle n|\right)}}
$$

with $c_{n}=(n-1) \Gamma\left(\frac{n-1}{2}\right) 2^{\frac{3 n-3}{2}} \pi^{\frac{n+1}{2}}$

By setting $\lambda=\langle H\rangle_{\rho_{0}}=\operatorname{tr}\left(\rho_{0} H\right)$ or $\lambda=E_{1}$, theorem 1 follows. On the other hand,

$$
\max _{\lambda}\left\{\sqrt{\left(E_{1}-\lambda\right)^{2} \operatorname{tr}\left(\rho_{0}|1\rangle\langle 1|\right)+\cdots+\left(E_{n}-\lambda\right)^{2} \operatorname{tr}\left(\rho_{0}|n\rangle\langle n|\right)}\right\}=\triangle E_{\rho_{0}}
$$

Thus, if

$$
t_{\text {exit }}(\epsilon) \leqslant \hbar \frac{\pi \cdot \min _{k}\left\{\sqrt{\operatorname{tr}\left(\rho_{0}|k\rangle\langle k|\right)}\right\}}{\triangle E_{\rho_{0}}}
$$

we can choose $\lambda$ such that

$$
t_{\text {exit }}(\epsilon)=\hbar \frac{\pi \cdot \min _{k}\left\{\sqrt{\operatorname{tr}\left(\rho_{0}|k\rangle\langle k|\right)}\right\}}{\sqrt{\left(E_{1}-\lambda\right)^{2} \operatorname{tr}\left(\rho_{0}|1\rangle\langle 1|\right)+\cdots+\left(E_{n}-\lambda\right)^{2} \operatorname{tr}\left(\rho_{0}|n\rangle\langle n|\right)}}
$$

and theorem 2 follows by inequality (21).

Proof of proposition 6. The recurrence time for the Hamiltonian $H$ is the same as the recurrence time for the zero-point rescaled Hamiltonian $H_{\lambda}=H-\lambda I$. Given an initial state $\rho_{0}$, by using equation (19), the temporal evolution is given by $\rho(t)=\pi\left(\mathrm{e}^{-\frac{\mathrm{it}}{\hbar}(H-\lambda I)} W\right)$, where $W=s\left(\rho_{0}\right)$. But given the basis $\{|k\rangle\}$ of eigenvalues for the Hamiltonian, 


$$
\mathrm{e}^{-\frac{\mathrm{it}}{\hbar}(H-\lambda I)} W=\mathrm{e}^{-\frac{\mathrm{it}}{\hbar}(H-\lambda I)} \sum_{k=1}^{n}|k\rangle\left\langle k\left|W=\sum_{k=1}^{n} \mathrm{e}^{-\frac{\mathrm{it}}{\hbar}\left(E_{k}-\lambda\right)}\right| k\right\rangle\langle k| W
$$

In this way, the curve $\gamma(t)=\mathrm{e}^{-\frac{\mathrm{it}}{\hbar}(H-\lambda I)} W$ is a curve in the torus

$$
\mathbb{T}^{n}(W):=\left\{\sum_{k=1}^{n} \mathrm{e}^{\mathrm{i} \theta_{k}}|k\rangle\langle k| W: \theta_{j} \in[0,2 \pi], \forall j\right\}
$$

We can make use of the following diffeomorphism

$$
\varphi: \mathbb{T}^{n} \rightarrow \mathbb{T}^{n}(W), \quad \varphi\left(\mathrm{e}^{\mathrm{i} \theta_{1}}, \cdots, \mathrm{e}^{\mathrm{i} \theta_{n}}\right)=\left(\sum_{k=1}^{n} \mathrm{e}^{\mathrm{i} \theta_{k}}|k\rangle\langle k| W\right)
$$

and the inclusion map $\mathbb{T}^{n}(W) \subset M_{n}(\mathbb{C})$ to pull back the metric from $M_{n}(\mathbb{C})$,

$$
\begin{aligned}
g\left(X_{j}, X_{l}\right)= & \frac{1}{2} \operatorname{tr}\left(e_{j}^{\dagger} e_{l}+e_{l}^{\dagger} e_{j}\right) \\
= & \frac{1}{2} \operatorname{tr}\left(W^{\dagger}|j\rangle\left\langle j\left|\mathrm{e}^{-\mathrm{i} \theta_{j}}(-\mathrm{i}) \mathrm{ie}^{\mathrm{i} \theta_{l}}\right| l\right\rangle\langle l| W\right. \\
& \left.+W^{\dagger}|l\rangle\left\langle l\left|\mathrm{e}^{-\mathrm{i} \theta_{l}}(-\mathrm{i}) \mathrm{ie}^{\mathrm{i} \theta_{j}}\right| j\right\rangle\langle j| W\right) \\
= & \delta_{j, l} \cdot \operatorname{tr}\left(\rho_{0}|j\rangle\langle j|\right)
\end{aligned}
$$

where $e_{j}=\mathrm{d} \varphi\left(X_{j}\right)$, and $\left\{X_{1}, \ldots, X_{n}\right\}$ is the basis of the Lie algebra $\mathfrak{t}^{n}$ (see appendix A.3) given by

$$
X_{j}=(\overbrace{0, \ldots, 0}^{j-1 \text { times }}, \mathrm{ie}^{\mathrm{i} \theta_{j}}, 0, \ldots, 0)
$$

The metric $g$ is a bi-invariant metric and since $\left[X_{j}, X_{l}\right]=0$, the torus $\left(\mathbb{T}^{n}, g\right)$ is a flat torus. In fact, geometrically $\left(\mathbb{T}^{n}, g\right)$ is the following torus

$$
\mathbb{T}^{n}:=\underset{k=1}{n} \mathbb{S}^{1}\left(\sqrt{\operatorname{tr}\left(\rho_{0}|k\rangle\langle k|\right)}\right)
$$

where $\mathbb{S}^{1}\left(\sqrt{\operatorname{tr}\left(\rho_{0}|k\rangle\langle k|\right)}\right)$ is the circle of radius $\sqrt{\operatorname{tr}\left(\rho_{0}|k\rangle\langle k|\right)}$. The injectivity radius (see proposition 9) is given by $\operatorname{inj}\left(\mathbb{T}^{n}\right)=\pi \cdot \min _{k}\left\{\sqrt{\operatorname{tr}\left(\rho_{0}|k\rangle\langle k|\right)}\right\}$. Furthermore, the curve $\widetilde{\gamma}=\varphi^{-1} \circ \gamma$ is a geodesic curve because it is the following curve

$$
\widetilde{\gamma}(t)=\left(\mathrm{e}^{-\mathrm{i} \frac{t}{\hbar}\left(E_{1}-\lambda\right)}, \cdots, \mathrm{e}^{-\mathrm{i} \frac{t}{\hbar}\left(E_{n}-\lambda\right)}\right)
$$

The length of $\widetilde{\gamma}([0, t])$ is given by

$$
\begin{aligned}
\operatorname{length}(\widetilde{\gamma}([0, t])) & =\int_{0}^{t} \sqrt{g(\dot{\tilde{\gamma}}(s), \dot{\tilde{\gamma}}(s))} \mathrm{d} s \\
& =\left\|-\frac{E_{1}-\lambda}{\hbar} X_{1}-\cdots-\frac{E_{n}-\lambda}{\hbar} X_{n}\right\| t \\
& =v t,
\end{aligned}
$$


where $v:=\sqrt{\left(\frac{E_{1}-\lambda}{\hbar}\right)^{2} \operatorname{tr}\left(\rho_{0}|1\rangle\langle 1|\right)+\cdots+\left(\frac{E_{n}-\lambda}{\hbar}\right)^{2} \operatorname{tr}\left(\rho_{0}|n\rangle\langle n|\right)}$. If $v \neq 0$, then for any $t<\frac{\operatorname{inj}\left(\mathbb{T}^{2}\right)}{v}$ we have $\operatorname{dist}_{\mathbb{T}^{n}}(\widetilde{\gamma}(0), \widetilde{\gamma}(t))=v t$.

Now we are going to define two different times for the movement in $\mathbb{T}^{n}$. Given $\delta>0$, we define the first exit time $t_{\text {exit }}^{\mathbb{T}^{n}}$ as the first time when $\operatorname{dist}_{\mathbb{T}^{n}}\left(\widetilde{\gamma}(0), \widetilde{\gamma}\left(t_{\text {exit }}^{\mathbb{T}^{n}}\right)\right)=\delta$. For the same $\delta>0$ we define the recurrence time $t_{\text {rec }}^{\mathbb{T}^{n}}$ as the first time after $t_{\text {exit }}^{\mathbb{T}^{n}}$ such that $\operatorname{dist}_{\mathbb{T}^{n}}\left(\widetilde{\gamma}(0), \widetilde{\gamma}\left(t_{\text {rec }}^{\mathbb{T}^{n}}\right)\right)=\delta$. Considering the following sequence of isometries $(\varphi)$, immersions $(i)$, and Riemannian submersions $(\pi)$

$$
\mathbb{T}^{n} \stackrel{\varphi}{\longrightarrow} \mathbb{T}^{n}(W) \stackrel{i}{\hookrightarrow} S \stackrel{\pi}{\longrightarrow} \mathcal{P}^{+}
$$

we conclude that

$$
\operatorname{dist}_{\mathbb{T}^{n}}(\widetilde{\gamma}(0), \widetilde{\gamma}(t)) \geqslant \operatorname{dist}_{\text {Bures }}\left(\rho_{0}, \rho(t)\right)=\sqrt{2-2 F\left(\rho_{0}, \rho(t)\right)}
$$

Therefore $t_{\text {exit }}\left(1-\frac{\delta^{2}}{2}\right) \geqslant t_{\text {exit }}^{\mathbb{T}^{n}}$ and if $t_{\text {exit }}\left(1-\frac{\delta^{2}}{2}\right)<t_{\text {rec }}^{\mathbb{T}^{n}}$ then $t_{\text {rec }}\left(1-\frac{\delta^{2}}{2}\right) \leqslant t_{\text {rec }}^{\mathbb{T}^{n}}$. Our approach is to make use of $t_{\text {rec }}^{\mathbb{T}^{n}}$ to obtain upper bounds for $t_{\mathrm{rec}}$. First of all we have to stress that if $\delta<\operatorname{inj}\left(\mathbb{T}^{n}\right)$ then

$$
\begin{aligned}
& t_{\mathrm{exit}}^{\mathbb{T}^{n}}=\frac{\delta}{v}, \\
& t_{\mathrm{rec}}^{\mathbb{T}^{n}} \geqslant \frac{\operatorname{inj}\left(\mathbb{T}^{n}\right)}{v}
\end{aligned}
$$

Now, we are going to obtain upper bounds for $t_{\text {rec }}^{\mathbb{I}^{n}}$ by using the volume of the tube $\widetilde{\gamma}^{\theta}([0, t])$. Recall that the tube $\widetilde{\gamma}^{\theta}([0, t])$ is the set of points of $\mathbb{T}^{n}$ which are at a distance of, at most $\theta$, through normal geodesics emanating from $\widetilde{\gamma}([0, t])$. To estimate the volume of such a tube, we first need to estimate the minimal focal distance of the tube.

The boundary of the tube $\partial \widetilde{\gamma}^{\theta}([0, t])$ with $\theta<\delta / 2$ and $\delta<\operatorname{inj}\left(\mathbb{T}^{n}\right)$ has no self-intersections for $t<t_{\text {rec }}^{\mathbb{T}^{n}}$. Otherwise, it would imply that there exist two times $t_{1}$ and $t_{2}$ with $0 \leqslant t_{1}<t_{2}<t_{\text {rec }}^{\mathbb{T}^{n}}$, such that two normal geodesics $\alpha, \beta$ to $\widetilde{\gamma}$ starting at $\widetilde{\gamma}\left(t_{1}\right)$ and at $\widetilde{\gamma}\left(t_{1}\right)$, respectively, coincide at some point of $\partial \widetilde{\gamma}^{\theta}([0, t])$. This means that

$$
\begin{array}{cccc}
\alpha(0)=\widetilde{\gamma}\left(t_{1}\right), & \dot{\alpha}(0)=Y, \quad \nabla_{\dot{\alpha}} \dot{\alpha}=0, & \|Y\|=1, & \langle Y, v\rangle=0 \\
\beta(0)=\widetilde{\gamma}\left(t_{2}\right), & \dot{\beta}(0)=Z, \quad \nabla_{\dot{\beta}} \dot{\beta}=0, & \|Z\|=1, & \langle Z, v\rangle=0 \\
\alpha(\theta)=\beta(\theta) &
\end{array}
$$

But this is a contradiction if $t_{2}<t_{\text {rec }}^{\mathbb{T}^{n}}$, because by the equation of the geodesics (see appendix A.3),

$\left(\mathrm{e}^{\mathrm{i}\left(Y_{1} \theta-\frac{E_{1}-\lambda}{\hbar} t_{1}\right)}, \cdots, \mathrm{e}^{\mathrm{i}\left(Y_{n} \theta-\frac{E_{n}-\lambda}{\hbar} t_{1}\right)}\right)=\left(\mathrm{e}^{\mathrm{i}\left(Z_{1} \theta-\frac{E_{1}-\lambda}{\hbar} t_{2}\right)}, \cdots, \mathrm{e}^{\mathrm{i}\left(Z_{n} \theta-\frac{E_{n}-\lambda}{\hbar} t_{2}\right)}\right)$

Hence,

$\left(\mathrm{e}^{\mathrm{i}\left(Y_{1}-Z_{1}\right) \theta}, \cdots, \mathrm{e}^{\mathrm{i}\left(Y_{n}-Z_{n}\right) \theta}\right)=\left(\mathrm{e}^{-\mathrm{i} \frac{E_{1}-\lambda}{\hbar}\left(t_{2}-t_{1}\right)}, \cdots, \mathrm{e}^{-\mathrm{i} \frac{E_{n}-\lambda}{\hbar}\left(t_{2}-t_{1}\right)}\right)=\widetilde{\gamma}\left(t_{2}-t_{1}\right)$ 
This means that there is a geodesic $c(t)$ of $\mathbb{T}^{n}$ which joins $\widetilde{\gamma}(0)$ and $\widetilde{\gamma}\left(t_{2}-t_{1}\right)$ with $c(0)=\widetilde{\gamma}(0)$, $c(\theta)=\widetilde{\gamma}\left(t_{2}-t_{1}\right), \dot{c}(t)=Y-Z$, and therefore

$$
\text { length }\left(c([0, \theta])=\|Y-Z\| \theta \leqslant(\|Y\|+\|Z\|) \theta=2 \theta<\delta<\operatorname{inj}\left(\mathbb{T}^{n}\right)\right.
$$

Then $\operatorname{dist}_{\mathbb{T}^{n}}\left(\widetilde{\gamma}(0), \widetilde{\gamma}\left(t_{2}-t_{1}\right)\right) \leqslant \delta$, hence $t_{2}-t_{1}<t_{\text {exit }}^{\mathbb{T}^{n}}$ and $v\left(t_{2}-t_{1}\right)<\delta$. Furthermore, $c([0, \theta])$ and $\widetilde{\gamma}\left(\left[0, t_{2}-t_{1}\right]\right)$ are two geodesics inside a geodesic ball centered at $\widetilde{\gamma}(0)$ of radius less than the injectivity radius of $\mathbb{T}^{n}$ and $c(\theta)=\widetilde{\gamma}\left(t_{2}-t_{1}\right)$ (and $c(0)=\widetilde{\gamma}(0)$ ), then $c([0, \theta])=\widetilde{\gamma}\left(\left[0, t_{2}-t_{1}\right]\right)$, but this is a contradiction because $c$ is normal to $\widetilde{\gamma}(\langle Y-Z, v\rangle=0)$.

Since $\widetilde{\gamma}$ is a geodesic of $\mathbb{T}^{n}$, and $\mathbb{T}^{n}$ is a flat manifold, there are no focal points along a geodesic normal to a geodesic of $\mathbb{T}^{n}$ (see [16, proposition 2.12]). We have proved that there are no overlaps, then

$$
\operatorname{minfoc}(\widetilde{\gamma}([0, t]))<\theta
$$

for any $t<t_{\text {rec }}^{\mathbb{T}^{n}}, \theta<\delta / 2, \delta<\operatorname{inj}\left(\mathbb{T}^{n}\right)=\pi \cdot \min _{k}\left\{\sqrt{\operatorname{tr}\left(\rho_{0}|k\rangle\langle k|\right)}\right\}$. Therefore, the $\theta$-tubular neighborhood $\widetilde{\gamma}^{\theta}([0, t])$ of $\widetilde{\gamma}([0, t])$ has volume (see $[15$, corollary 8.6])

$$
\mu\left(\widetilde{\gamma}^{\theta}([0, t])\right)=\frac{2 \pi^{\frac{n-1}{2}}}{(n-1) \Gamma\left(\frac{n-1}{2}\right)} \theta^{n-1} \cdot \operatorname{length}(\widetilde{\gamma}([0, t]))
$$

But using equality (23)

$$
\mu\left(\left(\gamma^{\theta}([0, t])\right)=\frac{2 \pi^{\frac{n-1}{2}}}{(n-1) \Gamma\left(\frac{n-1}{2}\right)} \theta^{n-1} v t .\right.
$$

Consequently, taking into account that $\mu\left(\widetilde{\gamma}^{\theta}\left(\left[0, t_{\text {rec }}^{\mathbb{T}^{n}}\right]\right) \leqslant \mu\left(\mathbb{T}^{n}\right)\right.$, we obtain

$$
t_{\mathrm{rec}}^{\mathbb{T}^{n}} \leqslant \frac{(n-1) \Gamma\left(\frac{n-1}{2}\right) 2^{n-1} \pi^{\frac{n+1}{2}} \prod_{k} \sqrt{\operatorname{tr}\left(\rho_{0}|k\rangle\langle k|\right)}}{\theta^{n-1} v}
$$

letting $\theta$ tend to $\delta / 2$,

$$
t_{\mathrm{rec}}^{\mathbb{T}^{n}} \leqslant \frac{(n-1) \Gamma\left(\frac{n-1}{2}\right) 2^{2 n-2} \pi^{\frac{n+1}{2}} \prod_{k} \sqrt{\operatorname{tr}\left(\rho_{0}|k\rangle\langle k|\right)}}{\delta^{n-1} v}
$$

And finally taking into account that $t_{\text {rec }}\left(1-\frac{\delta^{2}}{2}\right) \leqslant t_{\text {rec }}^{\mathbb{T}^{n}}$

$$
t_{\mathrm{rec}}\left(1-\frac{\delta^{2}}{2}\right) \leqslant \frac{(n-1) \Gamma\left(\frac{n-1}{2}\right) 2^{2 n-2} \pi^{\frac{n+1}{2}} \prod_{k} \sqrt{\operatorname{tr}\left(\rho_{0}|k\rangle\langle k|\right)}}{\delta^{n-1} v}
$$

setting $\epsilon=1-\frac{\delta^{2}}{2}$,

$$
t_{\mathrm{rec}}(\epsilon) \leqslant \frac{(n-1) \Gamma\left(\frac{n-1}{2}\right) 2^{\frac{3 n-3}{2}} \pi^{\frac{n+1}{2}} \prod_{k} \sqrt{\operatorname{tr}\left(\rho_{0}|k\rangle\langle k|\right)}}{(1-\epsilon)^{\frac{n-1}{2}} v}
$$




\section{Discussion}

We have presented several upper bounds for the Poincaré recurrence time of mixed states of discrete spectrum. Among the different ways of measuring the similarity or difference between the initial mixed state and the recurrent mixed stated we make use of the fidelity between the initial and recurrent density operators. In this sense we say that the state $\rho(t)$ has a recurrence time $t_{\text {rec }}(\epsilon)$ if $t_{\text {rec }}(\epsilon)$ is the first time after the first exit time when the fidelity between the initial state and $\rho\left(t_{\mathrm{rec}}(\epsilon)\right)$ is $\epsilon$, i.e. $F\left(\rho_{0}, \rho\left(t_{\mathrm{rec}}(\epsilon)\right)\right)=\epsilon$. In the case of a finite spectrum we obtain the following upper bounds

$$
t_{\mathrm{rec}}(\epsilon) \leqslant\left(\frac{c_{n}}{(1-\epsilon)^{\frac{n-1}{2}} n^{\frac{n}{2}}}\right) \frac{\hbar}{\triangle E_{\rho_{0}}}
$$

or

$$
t_{\mathrm{rec}}(\epsilon) \leqslant\left(\frac{c_{n}}{(1-\epsilon)^{\frac{n-1}{2}} n^{\frac{n}{2}}}\right) \frac{\hbar}{\sqrt{\operatorname{tr}\left(\rho_{0}\left(H-E_{1} I\right)^{2}\right)}}
$$

These results are completely analogous to the previous ones for pure states (estimates (3) and (4)), where the Poincaré time is related with the average of the gap energy (or the uncertainty in the energy). More generally, we provide an upper bound that depends on the dimension of the Hilbert space and on the first exit time but not on other characteristics of the specific Hamiltonian. This upper bound has the form

$$
t_{\text {rec }}(\epsilon) \leqslant C(n, \epsilon) t_{\text {exit }}(\epsilon)
$$

The movement of $\rho(t)$ returns infinitely many times close to $\rho_{0}$. Indeed, for any $s>0$ there exists a time $T \geqslant s$ such that

$$
F\left(\rho(T), \rho_{0}\right)>\epsilon
$$

with

$$
s \leqslant T \leqslant C(n, \epsilon) s .
$$

For the case of a discrete but non-finite spectrum we can recover the previous bounds for the recurrence time by using the relevant number of states. We show that, given any normalized mixed state $\rho$, the mixed state $\rho$ has $N$ relevant states with precision $\delta_{N}$ in the sense that it is always possible to construct a finite dimensional approximation $\sigma^{N}$ satisfying $\left\|\rho-\sigma^{N}\right\|_{2}^{2}=\delta_{N}$, and we can use the upper bounds for the finite dimensional and normalized mixed state $\widetilde{\sigma}^{N}=\frac{\sigma^{N}}{\operatorname{tr}\left(\sigma^{N}\right)^{\frac{1}{N}}}$ because

$$
\|\rho(t)-\rho(0)\|_{2} \leqslant 2 \sqrt{\delta_{N}}+2 \operatorname{tr}\left(\sigma^{N}(0)\right)^{\frac{1}{N}} \sqrt{1-F^{2}\left(\widetilde{\sigma}^{N}(0), \widetilde{\sigma}^{N}(t)\right)}
$$

The fidelity of $\widetilde{\sigma}^{N}$ therefore controls the recurrence of $\rho$. Moreover, $\delta_{N} \rightarrow 0$ and $\operatorname{tr}\left(\sigma^{N}\right) \rightarrow 1$ when $N \rightarrow \infty$.

To prove these upper bounds for the recurrence time, we use techniques from differential geometry, which, by using Uhlmann's principal fiber bundle, allow us to describe the space of mixed states and to relate the time of recurrence with the Bures distance, which is the geometric distance in the base manifold and is related with the Uhlmann fidelity.

At the classical limit, the quantum Poincare recurrence might be expected to become the classical one. But it should be noted that by taking the limit $\hbar \rightarrow 0$ in inequality (26) or in inequality (27) 


$$
t_{\mathrm{rec}}(\epsilon) \rightarrow 0, \quad \text { as } \quad \hbar \rightarrow 0 .
$$

Moreover, because there is no quantum speed limit in the classical world (see [17, 18, 21, 22]), we can let $t_{\text {exit }}$ tend to 0 in inequality (28), and therefore

$$
t_{\text {rec }}(\epsilon) \rightarrow 0 \text {. }
$$

Similarly to what happens with the quantum speed limit, the classical limit for the Poincaré recurrence is zero. This shows that this first recurrence is a purely quantum phenomenon (it is only a tremor from the classical point of view). Nevertheless, since inequality (29) depends only on the number of relevant states $N$, it remains unaltered by the limit $\hbar \rightarrow 0$. Hence this phenomenon survives up to the classical limit. Our conjecture is that the classical Poincaré recurrence time is related with this second recurrence and therefore with the number of relevant states (as a measure of the volume of the classical phase space).

This geometric approach could be useful to study the classical limit and in particular the classical limit to chaotic systems. This is because, in the general case, the recurrent time after the first recurrent time can be described by

$$
s \leqslant T \leqslant C(N, \epsilon) s, \quad s>t_{\mathrm{rec}}(\epsilon),
$$

where here $N$ is the number of relevant states and $F\left(\rho(T), \rho_{0}\right)>\epsilon$. The above inequality holds even at the limit $t_{\text {rec }}(\epsilon) \rightarrow 0$. Since $C(N, \epsilon)$ grows enormously with $N$, this might be an indication of the presence of an integrable system if the symmetries of the system reduce the number of relevant states. In the opposite direction, quantum systems which display quantum chaology would be more likely to visit every possible eigenstate of the system, and it is therefore expected that quantum chaotic states should have the largest recurrence time. In short, to describe quantum chaotic states we will need a large number of relevant states. Future work should therefore improve the constant $C(N, \epsilon)$ and also determine whether the number of relevant states $N$ can be reduced when physical symmetries are involved.

\section{Acknowledgments}

The research of the first author (VG) has been supported in part by Universitat Jaume I Research Program Project P1-1B2012- 18, and DGI-MINECO grant (FEDER) MTM2013-48371-C22-PDGI from the Ministerio de Ciencia e Innovación (MCINN), Spain. The research of the second author (JMS) was supported in part by DGI-MINECO grant (FEDER) MTM201348371-C2-2-PDGI from the Ministerio de Ciencia e Innovación (MCINN), Spain.

\section{Appendix}

\section{A.1. Poincaré recurrence for isometries in metric spaces of finite measure}

To prove theorem 3, we have used the following Poincaré recurrence theorem in metric spaces

Theorem 7. Let $(M, d, \mu)$ be a metric space $(M, d)$ with finite measure $\mu(M)<\infty$. Then for any volume-preserving isometry $T: M \rightarrow M$, any point $p \in M$, and any $r>0$ there exists $N_{r} \in \mathbb{N}$ such that the distance $d\left(p, T^{N_{r}}(p)\right)$ from $p$ to $T^{N_{r}}(p)=\overbrace{T \circ \cdots \circ T}^{N_{r} \text { times }}(p)$ is bounded from above by $r,\left(d\left(p, T^{N_{r}}(p)\right)<r\right)$, with $N_{r}$ satisfying the following inequality 


$$
1 \leqslant N_{r} \leqslant \frac{\mu(M)}{\mu\left(B_{r / 2}(p)\right)}
$$

where $B_{r / 2}(p)$ denotes the metric ball centered at $p \in M$ of radius $r / 2$. Namely,

$$
B_{r / 2}(p):=\left\{x \in M: d(p, x)<\frac{r}{2}\right\}
$$

Proof. To prove this theorem we first need the following lemma

Lemma 8. There exists $N_{r} \in \mathbb{N}$ with $1 \leqslant N_{r} \leqslant \frac{\mu(M)}{\mu\left(B_{r / 2}(p)\right)}$ such that

$$
T^{N_{r}}\left(B_{r / 2}(p)\right) \cap B_{r / 2}(p) \neq \emptyset
$$

Proof. Suppose that there exists $N \geqslant 1$ such that $T^{i}\left(B_{r / 2}(p)\right) \cap T^{j}\left(B_{r / 2}(p)\right)=\emptyset$ for all $i, j<N, i \neq j$, then

$$
\mu\left(\bigcup_{i=1}^{N} T^{i}\left(B_{r / 2}(p)\right)\right)=\sum_{i=1}^{N} \mu\left(T^{i}\left(B_{r / 2}(p)\right)\right) \leqslant \mu(M)
$$

But since $T$ is a volume-preserving transformation, $N \mu\left(B_{r / 2}(p)\right) \leqslant \mu(M)$. If we take $S$ as the first integer such that

$$
S>\frac{\mu(M)}{\mu\left(B_{r / 2}(p)\right)}, \quad\left(S=\left\lceil\frac{\mu(M)}{\mu\left(B_{r / 2}(p)\right)}\right\rceil\right) .
$$

then there must exist $0<1 \leqslant i<j \leqslant S$ such that $T^{i}\left(B_{r / 2}\right) \cap T^{j}\left(B_{r / 2}\right) \neq 0$, but taking $T^{-i}$ in this expression we obtain,

$$
B_{r / 2} \cap T^{j-i}\left(B_{r / 2}\right) \neq 0
$$

the lemma follows if we set $N_{r}=j-i$ and take into account that $1 \leqslant j-i \leqslant S-1$ and that $S \leqslant \frac{\mu(M)}{\mu\left(B_{r / 2}(p)\right)}+1$.

By applying the above lemma there exists $q \in B_{r / 2}$ such that $T^{N_{r}}(q) \in B_{r / 2}(p)$. This implies that $\mathrm{d}\left(p, T^{N_{r}}(q)\right) \leqslant \frac{r}{2}$ but since $T$ is an isometry $d\left(T^{N_{r}}(p), T^{N_{r}}(q)\right)=\mathrm{d}(p, q)$ and hence, by triangular inequality,

$$
d\left(p, T^{N_{r}}(p)\right) \leqslant d\left(p, T^{N_{r}}(q)\right)+d\left(T^{N_{r}}(p), T^{N_{r}}(q)\right) \leqslant \frac{r}{2}+\frac{r}{2}=r
$$

\section{A.2. Volume of balls in real space forms}

Let $\mathbb{M}_{\kappa}^{n}$ be the simply connected Riemannian manifold of constant sectional curvature $\kappa$ and dimension $n$. About each point $p \in \mathbb{M}_{\kappa}^{n}$ there exists a coordinate system $(t, \theta) \in[0, \pi / \sqrt{\kappa}] \times \mathbb{S}^{n-1}$, relative to which the Riemannian metric reads as (see [9, page 39])

$$
\mathrm{d} s^{2}=(\mathrm{d} t)^{2}+\mathrm{S}_{\kappa}^{2}(t)|\mathrm{d} \theta|^{2}
$$

where $S_{\kappa}(t)$ is the solution to the following differential equation with initial conditions 


$$
\mathrm{S}_{\kappa}^{\prime \prime}+\kappa \mathrm{S}_{\kappa}=0, \quad \mathrm{~S}_{\kappa}(0)=0, \quad \mathrm{~S}_{\kappa}^{\prime}(0)=1 .
$$

Observe that the $t$-curves are geodesics and in the particular case of spheres (spaces of $\kappa=1$ ),

$$
\mathrm{S}_{1}(t)=\sin (t)
$$

then, the Riemannian volume element is

$$
\mathrm{dV}=\sin ^{n-1}(t) \mathrm{dV}_{\mathbb{S}^{n-1}}
$$

where $\mathrm{d} V_{\mathbb{S}^{n-1}}$ is the Riemannian volume element in $\mathbb{S}^{n-1}$. The volume of the geodesic ball $B_{r}$ of radius $r$ in $\mathbb{S}_{1}^{n}$ can be obtained as

$$
\mathrm{V}\left(B_{r}\right)=\mathrm{V}\left(\mathbb{S}^{n-1}\right) \int_{0}^{r} \sin ^{n-1}(t) \mathrm{d} t=\frac{2 \pi^{n / 2}}{\Gamma(n / 2)} \int_{0}^{r} \sin ^{n-1}(t) \mathrm{d} t
$$

See also [15, page 252] for the general expression of the volume of a geodesic ball.

\section{A.3. The injectivity radius of a $n$-dimensional flat torus}

Let $\mathbb{T}^{n}=\overbrace{U(1) \times \ldots \times U(1)}^{n \text {-times }}$ denote the $n$-dimensional torus, that is,

$$
\mathbb{T}^{n}=\left\{\left(\mathrm{e}^{\mathrm{i} \theta_{1}}, \ldots, \mathrm{e}^{\mathrm{i} \theta_{n}}\right): \theta_{1}, \cdots, \theta_{n} \in \mathbb{R}\right\}
$$

with the usual product law

$$
\left(\mathrm{e}^{\mathrm{i} \theta_{1}}, \ldots, \mathrm{e}^{\mathrm{i} \theta_{n}}\right) \star\left(\mathrm{e}^{\mathrm{i} \alpha_{1}}, \ldots, \mathrm{e}^{\mathrm{i} \alpha_{n}}\right)=\left(\mathrm{e}^{\mathrm{i}\left(\theta_{1}+\alpha_{1}\right)}, \ldots, \mathrm{e}^{\mathrm{i}\left(\theta_{n}+\alpha_{n}\right)}\right) .
$$

Taking derivatives at $t=0$ in $\left(\mathrm{e}^{\mathrm{i} \theta_{1}}, \ldots, \mathrm{e}^{\mathrm{i} \theta_{n}}\right) \star\left(\mathrm{e}^{\mathrm{i} t}, 1, \ldots, 1\right)$ we obtain the left invariant vector field

$$
X_{1}=\left(\mathrm{ie}^{\mathrm{i} \theta_{1}}, 0, \ldots, 0\right)
$$

Similarly,

$$
X_{2}=\left(0, \mathrm{ie}^{\mathrm{i} \theta_{2}}, 0, \ldots, 0\right), \quad \cdots \quad X_{n}=\left(0,0 \cdots, 0, \mathrm{ie}^{\mathrm{i} \theta_{n}}\right)
$$

we obtain a basis $\left\{X_{1}, \cdots, X_{n}\right\}$ of the Lie algebra $\mathfrak{t}^{n}$ of $\mathbb{T}^{n}$. In fact, since the group is Abelian, $\left\{X_{1}, \cdots X_{n}\right\}$ are also right invariant vector fields. Now given an $n$-tuple of non-zero real numbers $\left(g_{1}, \ldots, g_{n}\right) \in \mathbb{R}^{n}$ we can define the bi-invariant metric

$$
g\left(X_{j}, X_{k}\right)=\delta_{j, k} g_{j}^{2}
$$

The sectional curvature $\kappa\left(X_{j}, X_{k}\right)$ of the plane spanned by $X_{j}$ and $X_{k}$ in a Lie group with biinvariant metric is given by (see [23]) $\kappa\left(X_{j}, X_{k}\right)=\frac{1}{4}\left\|\left[X_{j}, X_{k}\right]\right\|^{2}$. But, as can be easily checked, $\left[X_{j}, X_{k}\right]=0$, and therefore $\left(\mathbb{T}^{n}, g\right)$ is a flat torus. Moreover, as a Riemannian manifold $\left(\mathbb{T}^{n}, g\right)$ is isometric to

$$
\mathbb{R}^{n} / \Lambda=\left(\mathbb{S}^{1}, g_{1}^{2} \mathrm{~d} \theta_{1}^{2}\right) \times \cdots \times\left(\mathbb{S}^{1}, g_{n}^{2} \mathrm{~d} \theta_{n}^{2}\right)
$$

because

$$
\varphi: \mathbb{R}^{n} / \Lambda \rightarrow\left(\mathbb{T}^{n}, g\right), \quad\left(\theta_{1}, \ldots, \theta_{n}\right) \rightarrow \varphi\left(\theta_{1}, \ldots, \theta_{n}\right)=\left(\mathrm{e}^{\mathrm{i} \theta_{1}}, \ldots, \mathrm{e}^{\mathrm{i} \theta_{n}}\right)
$$


is a Riemannian isometry, since

$\mathrm{d} \varphi\left(v_{1} \partial \theta_{1}, \ldots v_{n} \partial \theta_{n}\right)=v_{1} X_{1}+\cdots+v_{n} X_{n}, \quad$ and $\quad \mathrm{d} s^{2}(u, v)=g(\mathrm{~d} \varphi(u), \mathrm{d} \varphi(v))$.

Then, we can obtain the volume of $\left(\mathbb{T}^{n}, g\right)$ as

$$
\operatorname{vol}\left(\mathbb{T}^{n}, g\right)=(2 \pi)^{n} \prod_{j=1}^{n}\left|g_{j}\right|
$$

and the geodesic curves starting at $p=\left(\mathrm{e}^{\mathrm{i} \theta_{1}}, \ldots, \mathrm{e}^{\mathrm{i} \theta_{n}}\right)$ with tangent vector $v \in T_{p} \mathbb{T}^{n}$, $v=v_{1} X_{1}(p)+\cdots v_{n} X_{n}(p)$ are given by (see [24, corollary 57])

$$
\gamma(t)=\left(\mathrm{e}^{\mathrm{i} v_{1} t}, \ldots, \mathrm{e}^{\mathrm{i} v_{n} t}\right) \star\left(\mathrm{e}^{\mathrm{i} \theta_{1}}, \ldots, \mathrm{e}^{\mathrm{i} \theta_{n}}\right)
$$

and moreover we can obtain the injectivity radius.

Proposition 9. The injectivity radius $\operatorname{inj}\left(\mathbb{T}^{n}, g\right)$ of $\left(\mathbb{T}^{n}, g\right)$ is given by

$$
\operatorname{inj}\left(\mathbb{T}^{n}, g\right)=\pi \min _{j \in\{1, \ldots, n\}}\left\{\left|g_{j}\right|\right\}
$$

Proof. Since $\left(\mathbb{T}^{n}, g\right)$ is a flat manifold, the injectivity radius is given (see [28, corollary 4.14 of chapter III]) by half the length of the shortest closed non-trivial geodesic. But since $\left(\mathbb{T}^{n}, g\right)$ is isometric to $\mathbb{R}^{n} / \Lambda$, and $\mathbb{R}^{n} / \Lambda$ is a product manifold, this length is the length of the shortest closed geodesic of one of the factors (see [24, corollary 57]).

\section{References}

[1] Arnol'd V I 1989 Mathematical Methods of Classical Mechanics (Graduate Texts in Mathematics vol 60) (New York: Springer) (translated from the 1974 Russian original by K Vogtmann and A Weinstein, corrected reprint of the second (1989) edition)

[2] Barbón J L F and Rabinovici E 2003 J. High Energy Phys. JHEP7(2003)1073-99

[3] Bhattacharyya K and Mukherjee D 1986 J. Chem. Phys. 84 3212-4

[4] Bengtsson I and Życzkowski K 2006 Geometry of Quantum States (Cambridge: Cambridge University Press) (an introduction to quantum entanglement)

[5] Berman G P, Bishop A R, Borgonovi F and Dalvit D A R 2004 Phys. Rev. A 69 062110-1

[6] Bocchieri P and Loinger A 1957 Phys. Rev. 107 337-8

[7] Castagnino M and Fortin S 2011 Int. J. Theor. Phys. 50 2259-67

[8] Chandrashekar C M 2010 Cent. Eur. J. Phys. 8 976-8

[9] Chavel I 1984 Eigenvalues in Riemannian Geometry (Pure and Applied Mathematics vol 115) (Orlando, FL: Academic Press Inc.) (including a chapter by Burton Randol, with an appendix by Jozef Dodziuk)

[10] Chruściński D and Jamiołkowski A 2004 Geometric Phases in Classical and Quantum Mechanics (Progress in Mathematical Physics vol 36) (Boston, MA: Birkhäuser Boston Inc.)

[11] Dąbrowski L and Jadczyk A 1989 J. Phys. A: Math. Gen. 22 3167-70

[12] Dąbrowski L and Grosse H 1990 Lett. Math. Phys. 19 205-10

[13] Dąbrowski L 1991 Nuovo Cimento B 106 963-8

[14] Dyson L, Kleban M and Susskind L 2002 J. High Energy Phys. JHEP11(2002)21

[15] Gray A 2004 Tubes 2nd edn (Progress in Mathematics vol 221) (Basel: Birkhäuser Verlag) (with a preface by Vicente Miquel)

[16] Gimeno V and Palmer V 2017 Mean curvature, volume and properness of isometric immersions 369 4347-66

[17] Giovanetti V, Lloyd S and Maccone L 2003 Phys. Rev. A 67052109

[18] Giovanetti V, Lloyd S and Maccone L 2004 J. Opt. G 6 S807

[19] Hogg T and Huberman B A 1982 Phys. Rev. Lett. 48 711-4

[20] Kaminishi E, Sato J and Deguchi T 2015 J. Phys. Soc. Japan 84064002 
[21] Margolus N and Levitin L B 1998 Physica D 120 188-95

[22] Mandelshtam L I and Tamm I E 1945 Acad. Sci. USSR J. Phys. 9 249-54

[23] Milnor J 1976 Adv. Math. 21 293-329

[24] O’Neill B 1983 Semi-Riemannian Geometry (Pure and Applied Mathematics vol 103) (New York: Academic) (with applications to relativity)

[25] Percival I C 1961 J. Math. Phys. 2 235-9

[26] Peres A 1982 Phys. Rev. Lett. 491118

[27] de Ponte M A, Cacheffo A, Villas-Bas C J, Mizrahi S S and Moussa M H Y 2010 Eur. Phys. J. D 59 487-96

[28] Sakai T 1996 Riemannian Geometry (Translations of Mathematical Monographs vol 149) (Providence, RI: American Mathematical Society) (translated from the 1992 Japanese original by the author)

[29] Schulman L S 1978 Phys. Rev. A 18 2379-80

[30] Siopsis G 2007 Class. Quantum Grav. 24 4133-45

[31] Uhlmann A 1986 Rep. Math. Phys. 24 229-240

[32] Uhlmann A 1989 Ann. Phys. 46 63-9

[33] Uhlmann A 1991 Lett. Math. Phys. 21 229-36

[34] Venugopalan A, Kumar D and Ghosh R 1995 Phys. A: Stat. Mech. Appl. 220 563-75

[35] Venuti L C 2015 arXiv:1509.04352

[36] Watrous J 2008 Theory of Quantum Information (Waterloo: University of Waterloo) www. cs.uwaterloo.ca/ $\sim$ watrous/quant-info/

[37] Zurek W H 1982 Phys. Rev. D 26 1862-80 